\title{
Mutations of EXOSC3/Rrp40p associated with neurological diseases impact ribosomal RNA processing functions of the exosome in $S$. cerevisiae
}

\author{
ABBY GILLESPIE, ${ }^{1,4}$ JASON GABUNILAS, ${ }^{1}$ JOANNA C. JEN, ${ }^{2,3}$ and GUILLAUME F. CHANFREAU ${ }^{1}$ \\ ${ }^{1}$ Department of Chemistry and Biochemistry and the Molecular Biology Institute, University of California, Los Angeles, California 90095-1569, USA \\ ${ }^{2}$ Department of Neurology, ${ }^{3}$ Department of Neurobiology, University of California, Los Angeles, California 90095-1763, USA
}

\begin{abstract}
The RNA exosome is a conserved multiprotein complex that achieves a large number of processive and degradative functions in eukaryotic cells. Recently, mutations have been mapped to the gene encoding one of the subunits of the exosome, EXOSC3 (yeast Rrp40p), which results in pontocerebellar hypoplasia with motor neuron degeneration in human patients. However, the molecular impact of these mutations in the pathology of these diseases is not well understood. To investigate the molecular consequences of mutations in EXOSC3 that lead to neurological diseases, we analyzed the effect of three of the mutations that affect conserved residues of EXOSC3/Rrp40p (G31A, G191C, and W238R; G8A, G148C, and W195R, respectively, in human and yeast) in $S$. cerevisiae. We show that the severity of the phenotypes of these mutations in yeast correlate with that of the disease in human patients, with the W195R mutant showing the strongest growth and RNA processing phenotypes. Furthermore, we show that these mutations affect more severely pre-ribosomal RNA processing functions of the exosome rather than other nuclear processing or surveillance functions. These results suggest that delayed or defective pre-rRNA processing might be the primary defect responsible for the pathologies detected in patients with mutations affecting EXOSC3 function in residues conserved throughout eukaryotes.
\end{abstract}

Keywords: pontocerebellar hypoplasia; exosome; EXOSC3; Rrp40; ribosomal RNA processing

\section{INTRODUCTION}

The RNA exosome is a multiprotein complex first identified in S. cerevisiae (Mitchell et al. 1997) and conserved in eukaryotic cells (Allmang et al. 1999b). This complex exhibits $3^{\prime}-5^{\prime}$ exoribonuclease activity, as well as endonuclease activity mediated by the Dis3p/Rrp44p subunit of the complex (Lebreton et al. 2008; Schaeffer et al. 2009; Schneider et al. 2009). The Dis3p/Rrp44p catalytic subunit associates with a core ring of six subunits and with three additional subunits to form a catalytically active complex (Wasmuth et al. 2014; Makino et al. 2015; Zinder et al. 2016). This complex is found both in the nucleus and cytoplasm of eukaryotic cells (Allmang et al. 1999a) and is involved in a variety of RNA processing and degradation functions. The major quantitative function of the exosome in eukaryotic cells is to process the precursor of ribosomal RNA by trimming some of the $3^{\prime}$ extensions of the pre-rRNAs that remain following some of the cleavage events (Allmang et al. 1999a). The nuclear exo-

${ }^{4}$ Present address: UCSD, 9500 Gilman Dr, La Jolla, CA 92093, USA Corresponding author: guillom@chem.ucla.edu

Article is online at http://www.rnajournal.org/cgi/doi/10.1261/rna.060004. 116. Freely available online through the RNA Open Access option. some also processes the precursor species of various small nuclear and nucleolar RNAs to generate their mature $3^{\prime}$ ends (Allmang et al. 1999a). In addition to these processing functions, the nuclear exosome of $S$. cerevisiae also degrades a large number of cryptic transcripts or precursor species (Kadaba et al. 2004; Wyers et al. 2005; Gudipati et al. 2012) and is involved in the quality control of gene expression by degrading unspliced RNAs in the nucleus (BousquetAntonelli et al. 2000; Sayani and Chanfreau 2012). The cytoplasmic exosome has been implicated in a variety of degradative functions, including surveillance of transcripts that have escaped from the nucleus and are defective for translation termination (van Hoof et al. 2002).

In higher eukaryotes, RNA degradative functions of the exosome have started to emerge due to studies that have analyzed the phenotypes of cells depleted for various components of the exosome (Lubas et al. 2015; Pefanis et al. 2015). Recently, mutations affecting EXOSC3, the human homolog of Rrp40p, were shown to cause pontocerebellar hypoplasia

(C) 2017 Gillespie et al. This article, published in $R N A$, is available under a Creative Commons License (Attribution 4.0 International), as described at http://creativecommons.org/licenses/by/4.0/. 
A

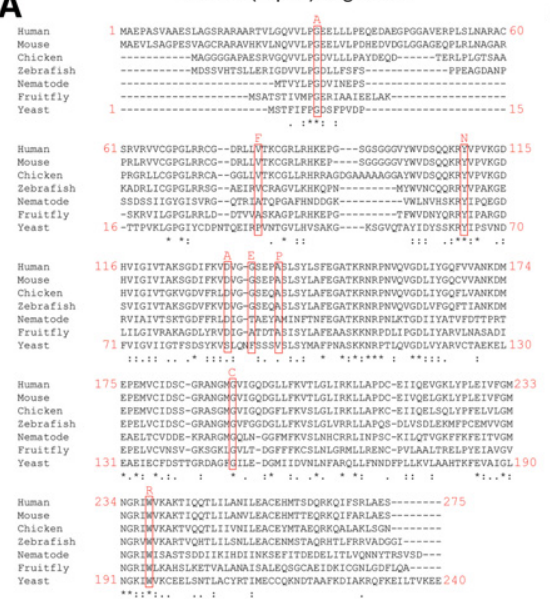

B

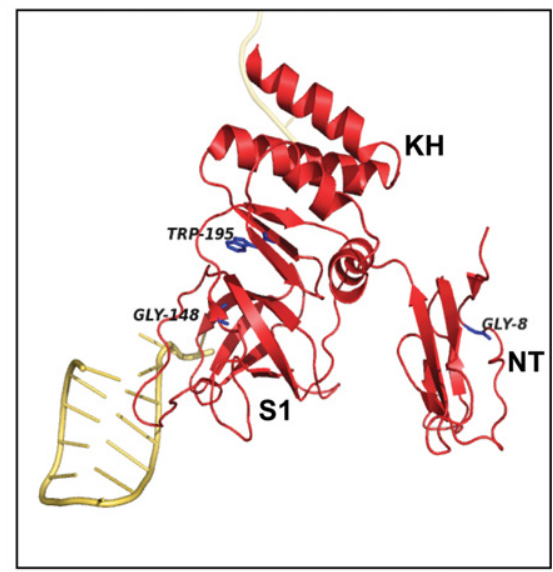

C
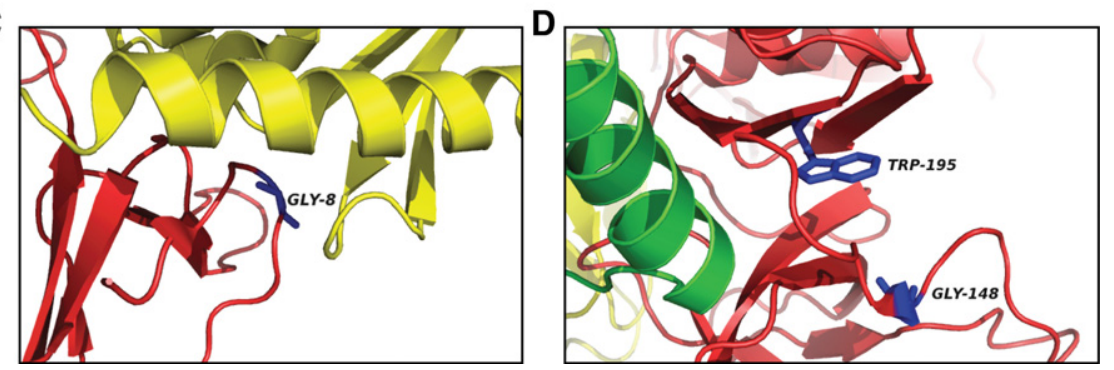

FIGURE 1. EXOSC3/Rrp40p mutations and location of the corresponding amino acids in Rrp40p structure. (A) Sequence alignment showing the position of mutations mapped in EXOSC 3 and the conservation of the corresponding amino acids in eukaryotic EXOSC3 homologs. The positions of the amino acids are indicated at the beginning and end of each line for the human and yeast sequences. (B) Structure of Rrp40p in the context of the global structure of the exosome. The locations of the three residues analyzed and of the main protein domains are indicated. The yellow strand indicates the RNA substrate. Residues analyzed in this study are highlighted in blue. The figure was generated using PyMol from PDB entry 4IFD (Makino et al. 2013). (C) Close view of the atomic environment of Rrp40p Gly8. Rrp40p is shown in red; Rrp46p is shown in yellow. Gly8 is highlighted in blue. The figure was generated using PyMol from PDB entry 4IFD (Makino et al. 2013). (D) Close view of the atomic environment of Rrp40p Gly148 and Trp195. Rrp40p is shown in red. The green a helix shown is part of Rrp45p. Gly148 and Trp195 are highlighted in blue. The figure was generated using PyMol from PDB entry 4IFD (Makino et al. 2013).

type 1B (PCH1B, OMIM614678) (Wan et al. 2012). To date, biallelic EXOSC3 mutations have been found in $~ 50 \%$ of patients with $\mathrm{PCH} 1$, which is characterized by maldevelopment and degeneration of cerebellar and spinal motor neurons (Wan et al. 2012; Rudnik-Schöneborn et al. 2013; Eggens et al. 2014). Disease-causing mutations reside in various functional domains of $\mathrm{EXOSC} 3$, ranging from the putative $\mathrm{KH}$ RNA binding domain to the interface with the rest of the complex (Fig. 1). Different mutations appear correlated with different severity in clinical manifestations and survival (Wan et al. 2012; Rudnik-Schöneborn et al. 2013; Eggens et al. 2014). Thus, the precise impact of the mutations on the function of the human exosome remains enigmatic, and how these mutations lead to human disease remains unclear. To investigate the molecular impact of disease-causing mutations on the function of the exosome, we introduced several corresponding mutations into the Rrp40p subunit of $S$. cerevisiae and analyzed the RNA processing and degradation phenotypes of these mutants in vivo.

\section{RESULTS AND DISCUSSION}

\section{The W195R mutation (EXOSC3 W238R) exhibits the most severe growth phenotype when introduced into Rrp40p}

To gain further insights into the potential mechanisms by which EXOSC3 mutations cause $\mathrm{PCH} 1 \mathrm{~B}$ and related disorders, we introduced some of these mutations into the $S$. cerevisiae homolog of EXOSC3, Rrp40p. Among all the mutations implicated in these pathologies, we chose to study three mutations that affect residues conserved in $S$. cerevisiae Rrp40p (Fig. 1A): G31A, G191C, and W238R. This choice was based on the consideration that mutations that affect residues conserved throughout evolution are likely to affect fundamental aspects of the structure or function of the eukaryotic exosome. In addition, these mutations are associated with two distinct pathologies linked to EXOSC3 defects involving motor neurons (Halevy et al. 2014), suggesting that they may impact the function of the exosome in cerebellar and motor neurons in a fundamental manner. G31 (G8 in Rrp40p) resides in a hydrophobic pocket located near the interface with EXOSC5 (Rrp46p in yeast; Fig. 1B,C; Makino et al. 2013). Thus the G31A/ G8A mutation might affect the interaction of EXOS3/Rrp40p with EXOSC5/Rrp46p (Fig. 1C) and thus the association of EXOSC3/Rrp40p with the exosome complex. W238 (W195 in S. cerevisiae) is located in the putative KH RNA binding domain of EXOSC3/Rrp40p (Fig. 1B), suggesting that mutations affecting this residue could impact RNA binding. In addition, W195 and G148 are located in close vicinity in the 3D structure of Rrp40p (Fig. 1D; Makino et al. 2013), suggesting that they might interact or cooperate to promote the structural or functional integrity of EXOSC3/Rrp40p and/or RNA binding (Fig. 1B).

In order to avoid potential dosage effects, these three mutations were introduced by integration into the endogenous chromosomal copy of RRP40 using the CRISPR system (Stovicek et al. 2015), rather than through extrachromosomal plasmid-borne copies. Accordingly, mutant versions of Rrp40p are expressed from the endogenous promoter and maintain identical $5^{\prime}$ and $3^{\prime}$ UTR sequences. After 


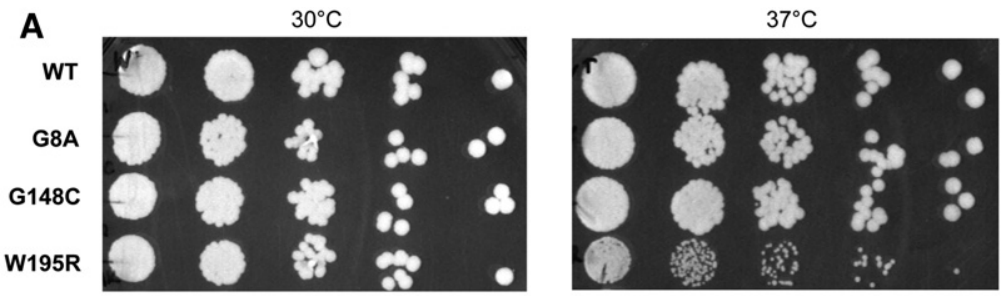

B

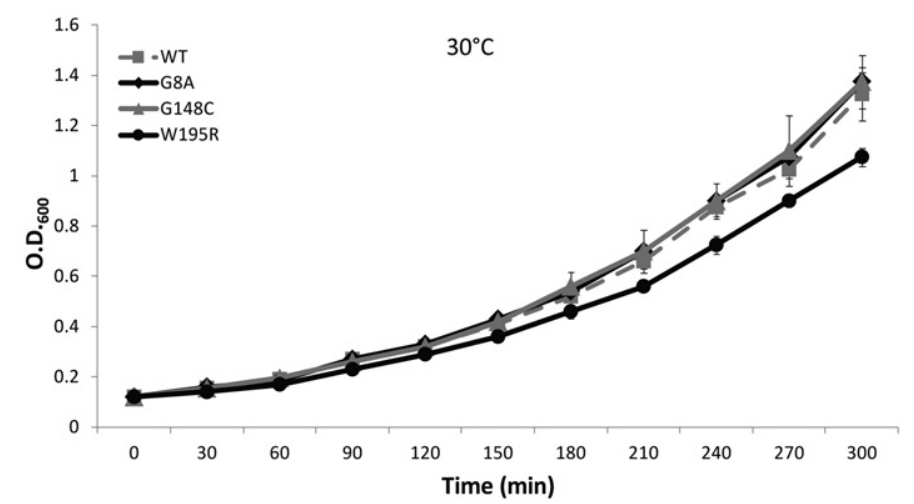

C

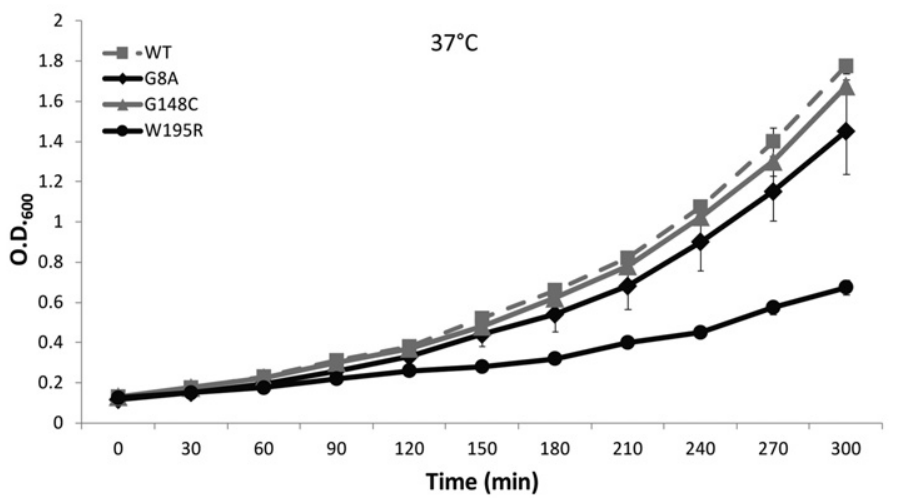

FIGURE 2. Growth of wild-type and Rrp40p mutants at $30^{\circ}$ and $37^{\circ} \mathrm{C}$. (A) Growth on solid medium at $30^{\circ} \mathrm{C}$ and $37^{\circ} \mathrm{C}$. Serial dilutions of liquid cultures were spotted on YPD and grown at the indicated temperatures. (B) Growth on liquid medium at $30^{\circ} \mathrm{C}$ and $37^{\circ} \mathrm{C}$. Shown are the average values and standard deviations observed for growth curves for two replicates performed simultaneously for each strain in the same batch of YPD medium and in the same incubator.

introduction of each mutation at the chromosomal RRP40 locus and confirmation by sequencing, growth of the mutant strains was compared to that of the isogenic wild-type strain at $30^{\circ} \mathrm{C}$ and $37^{\circ} \mathrm{C}$, both in solid and liquid media. Analysis of the growth of these mutants on YPD plates by serial spot dilution showed no obvious growth defect on rich medium at $30^{\circ} \mathrm{C}$, but the $\mathrm{W} 195 \mathrm{R}$ mutation resulted in a detectable growth impairment at $37^{\circ} \mathrm{C}$ (Fig. $2 \mathrm{~A}$ ). In contrast, the G8A and G148C mutants did not exhibit detectable growth delay on solid medium. However, because cell density on solid media can mask subtle growth phenotypes, we also assessed mutant strains growth in liquid medium at both temperatures (Fig. 2B). Analysis of growth curves performed in simultaneous biological replicates performed in the same batch of medium and in the same incubator confirmed the severe growth defect of the W195R mutant at $37^{\circ} \mathrm{C}$ and also revealed a significant growth defect for the W195R mutant at $30^{\circ} \mathrm{C}$ compared to the wildtype strain. In addition, while the G8A and G148C mutants grew similarly to wild type at $30^{\circ} \mathrm{C}$, their growth was clearly delayed compared to the wild-type reference strain at $37^{\circ} \mathrm{C}$. Similar growth defects were observed in a separate independent experiment (Supplemental Fig. S1). In conclusion, all of the mutations studied here that result in PCH1b or hereditary spastic paraplegia cause detectable growth defects when transposed into yeast Rrp40p, with the W195R causing the most severe growth phenotype.

\section{Mutations of Rrp40p have a modest impact on snRNA processing and degradative functions of the exosome}

To investigate the molecular effects of Rrp40p mutations, we chose to analyze a subset of exosome substrates by Northern blot analysis, as this technique provides the best way to specifically detect unprocessed species and intermediates, and to visualize the ratio of these species to mature RNAs. We chose to use as a loading control the 5S rRNA, since it was shown to be unaffected by various mutations affecting exosome function (Gudipati et al. 2012). The exosome is involved in several 3 '-end processing steps during processing of the ribosomal RNA precursor and also processes the $3^{\prime}$-ends of several small nuclear RNAs (Allmang et al. 1999a; van Hoof et al. 2000). In addition, the exosome promotes the degradation of unspliced RNAs (BousquetAntonelli et al. 2000; Sayani and Chanfreau 2012) and of many precursor RNA species, including tRNAs (Allmang et al. 2000; Gudipati et al. 2012). Northern blot analysis of the U4 snRNA, whose precursor is cleaved by Rntlp and then trimmed by the exosome (van Hoof et al. 2000), showed that the W195R mutation had the most severe impact on U4 snRNA processing, with an accumulation of the $3^{\prime}$-extended form of $\mathrm{U} 4$ at $37^{\circ} \mathrm{C}$ (Fig. 3A). Comparison with a sample generated from the Rrp6p knockout in which the 3'-extended form of U4 accumulates (Egecioglu et al. 2006) showed that this intermediate accumulated to levels slightly lower than in the sample from the $r r p 6 \Delta$ strain when strains were grown at $37^{\circ} \mathrm{C}$, suggesting partial defects in the exosome function. In addition, we also detected an increase of the 

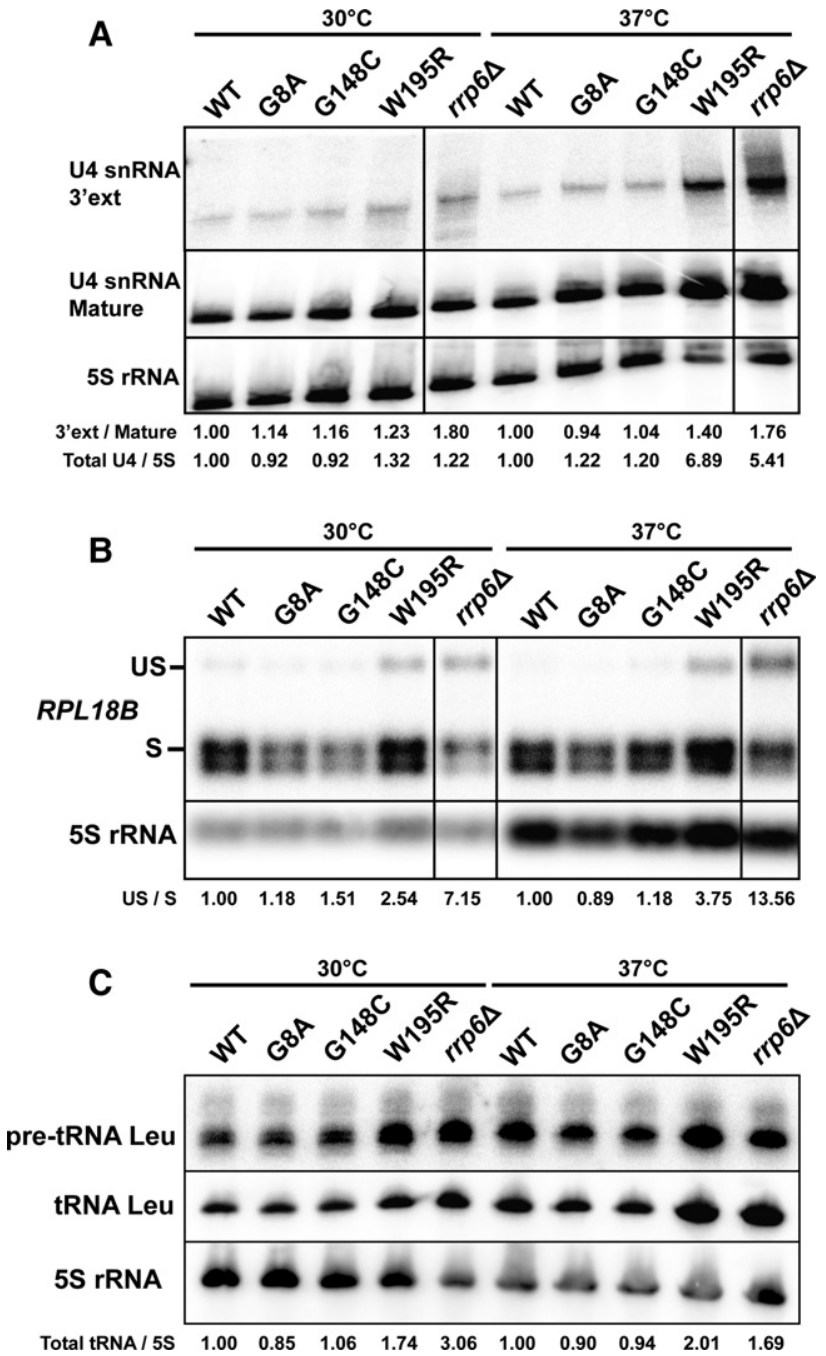

FIGURE 3. snRNA processing and degradation defects of Rrp40p mutants. (A) Analysis of U4 snRNA processing. Membranes were hybridized with an oligonucleotide probe downstream from the U4 snRNA or to an oligonucleotide hybridizing to the mature U4 snRNA. Lines drawn on the Northern blots indicate that lanes from these blots were removed from the picture. However, all the lanes shown for each panel originate from the same membranes and hybridizations. The 5S rRNA was used as a loading control. The values shown indicate the ratio of U4 $3^{\prime}$-extended species to mature species, normalized to that ratio for the respective WT sample at each temperature. Also provided are the total U4 signals normalized to the $5 \mathrm{~S}$ rRNA signals, which are in turn normalized to that ratio for the respective WT sample at each temperature. (B) Analysis of RPL18B spliced and unspliced RNA levels. The 5S rRNA was used as a loading control. Quantitation of unspliced to spliced (US/S) RPL18B was performed similarly to $A$. (C) Analysis of Leu pre-tRNA and tRNA levels. The $5 \mathrm{~S}$ rRNA was used as a loading control. Quantitation of total tRNA signals was performed similarly to $A$ and $B$.

levels of mature U4 in the W195R and rrp6 $\Delta$ strains (Fig. 3A). This increase might be due to reduced degradation of the $\mathrm{U} 4$ precursor by the exosome in these strains, as it was shown previously that the exosome degrades many precursor species of stable RNAs (Gudipati et al. 2012). The two other mutations did not exhibit any significant accumulation of 3 '-ex- tended U4 snRNA at any of the growth temperatures analyzed (Fig. 3A).

The nuclear exosome also degrades a large number of nuclear transcripts, including hypomodified tRNAs (Kadaba et al. 2004), CUTs, and many precursor species (Allmang et al. 2000; Gudipati et al. 2012). To investigate the impact of Rrp40p mutations on the exosome surveillance functions, we analyzed the accumulation of unspliced species of RPL18B and of Leu pre-tRNA, both of which were previously shown to be degraded by the exosome (Gudipati et al. 2012; Sayani and Chanfreau 2012). The W195R mutation exhibited an accumulation of unspliced RPL18B when this mutant was grown at either $30^{\circ} \mathrm{C}$ or $37^{\circ} \mathrm{C}$, indicative of defective surveillance functions similar to or slightly milder than those detected in the $r r p 6 \Delta$ strain (Fig. 3B). Analysis of the Leu tRNA precursors and mature species also showed an accumulation of precursor species in the $\mathrm{W} 195 \mathrm{R}$ mutant at $30^{\circ} \mathrm{C}$ and $37^{\circ} \mathrm{C}$. At $37^{\circ} \mathrm{C}$ this mutant also exhibited elevated levels of mature Leu tRNA (Fig. 3C), comparable to those detected in the $r r p 6 \Delta$ strain. This result is consistent with previous observations showing that degradation of pre-tRNA precursors by the exosome titrate these species away from the processing pathway (Gudipati et al. 2012), and that perturbation of exosome function-either by Rrp6p inactivation, or by the W195R mutation-increases tRNA levels by redirecting more precursors into the processing pathway, in a manner similar to what we observed previously for U4 (Fig. 3A). The other two RRP40 mutations did not exhibit any detectable phenotypes using these three exosome substrates, suggesting that their modest growth defect at $37^{\circ} \mathrm{C}$ (Fig. $2 \mathrm{~B}$ ) is due to other processing or degradation defects.

\section{Mutations of Rrp40p dramatically impair pre-rRNA processing}

We next analyzed the impact of the G8A, G148C, and W195R mutations on pre-rRNA processing by Northern blot analysis using probes hybridizing to both internal transcribed spacer (ITS1 and ITS2) sequences of the pre-rRNA precursor (Fig. 4A,B; Supplemental Fig. S2 shows a schematic representation of the pre-rRNA processing pathway with the location of the probes used). This analysis revealed that the W195R mutation resulted in a strong accumulation of the $35 \mathrm{~S}$ precursor at $37^{\circ} \mathrm{C}$ (Fig. $4 \mathrm{~A}, \mathrm{~B}$ ). In addition, this mutant showed reduced accumulation of the $20 \mathrm{~S}$ and $23 \mathrm{~S}$ species at $37^{\circ} \mathrm{C}$ (Fig. $4 \mathrm{~A}$ ). Since the production of the $20 \mathrm{~S}$ does not involve any exonuclease trimming (Supplemental Fig. S2), reduction of the levels of this intermediate in the W195R mutant is likely the result of an indirect effect and indicative of a general delay in pre-rRNA processing, consistent with pre-35S accumulation. The $\operatorname{rrp} 6 \Delta$ strain showed the accumulation of $23 \mathrm{~S}, 17 \mathrm{~S}^{\prime}$, and $\left(^{*}\right)$ species (Fig. 4A; species described in Supplemental Fig. S2) reported previously (Wery et al. 2009). Interestingly, the G8A and G148C mutants exhibited increased levels of the 23S (Fig. 4A), indicative of defective 

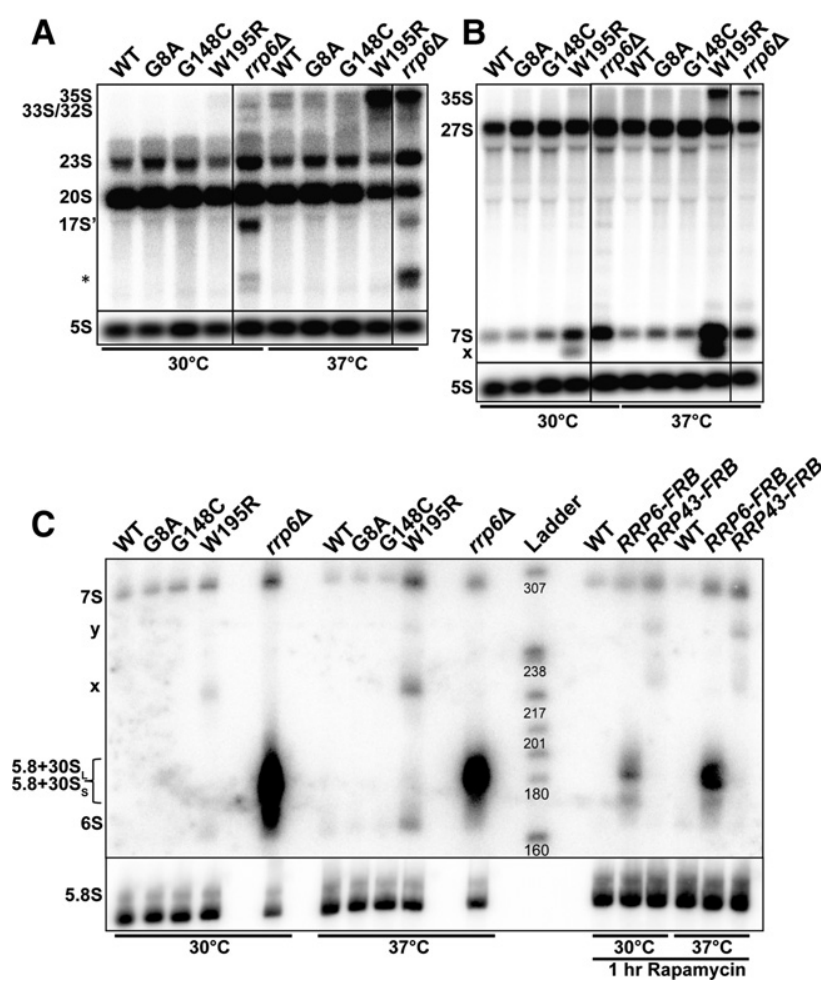

FIGURE 4. rRNA Processing analysis of Rrp40p mutants. (A) Agarose Northern blot analysis of precursor and intermediates containing the ITS1 sequence. Membranes were probed with probe O1663 (Supplemental Fig. S2) hybridizing to ITS1. The main precursors and intermediates containing the ITS1 sequence are labeled on the left. The species labeled $17 \mathrm{~S}^{\prime}$ and $\left({ }^{*}\right)$ are species that are detected specifically in the rrp6 6 mutant strain (Wery et al. 2009). The latter species is not well characterized but has been detected previously (Wery et al. 2009). (B) Agarose Northern blot analysis of precursor and intermediates containing the ITS2 sequence. Membranes were probed with probe O1660 (Supplemental Fig. S2) hybridizing to the ITS2 spacer. The main precursors and intermediates containing the ITS2 sequence are labeled on the left. The species labeled with " $\mathrm{x}$ " are the ones that accumulate specifically in the W195R mutant. (C) Acrylamide Northern blot analysis of precursor and intermediates containing the ITS2 sequence. Membranes were probed with probe 020 hybridizing to the ITS2 spacer (Supplemental Fig. S2). The main precursors and intermediates containing the ITS2 sequence are labeled on the left. The species labeled with " $\mathrm{x}$ " are the same as the ones highlighted in panel $B$. The species labeled with " $y$ " accumulates only in the W195R mutant and in the Rrp43 anchor away strain. The Rrp6 and Rrp43 anchor away strains and corresponding control strain were treated with Rapamycin to induce export of FRB-tagged proteins out of the nucleus.

processing or degradation of this intermediate. Hybridization with an ITS2 probe showed that the W195R mutation resulted in a severe accumulation of the 7S intermediate, particularly at $37^{\circ} \mathrm{C}$, which reflects a delay in the processing leading to the production of the $5.8 \mathrm{~S}$ rRNA (Fig. 4B). In addition to the accumulation of the $7 \mathrm{~S}$ intermediate, we also detected the accumulation of faster migrating species, which might correspond to processing intermediates between the $7 \mathrm{~S}$ and 5.8S rRNA (Fig. 4B). Interestingly, the G8A and G148C mutants also exhibited a slight but detectable accumulation of the $7 \mathrm{~S}$ intermediate, especially at $37^{\circ} \mathrm{C}$, indicative of a processing delay of the $5.8 \mathrm{~S}$ rRNA in these mutants. This phenotype, combined with the $23 \mathrm{~S}$ accumulation described above might be responsible for the slight growth defect detected in these mutants (Fig. 2B).

To gain a better resolution onto the processing defects of the 5.8S rRNA, we repeated this Northern blot analysis on an acrylamide gel, which allowed for a better separation of these species (Fig. 4C). This analysis confirmed the accumulation of the 7S intermediate in the W195R mutant, particularly at $37^{\circ} \mathrm{C}$. In addition, it also showed the appearance of processing intermediates, which migrate between the $7 \mathrm{~S}$ pre-rRNA and the 5.8S rRNA. These intermediates are distinct from the extended species of the $5.8 \mathrm{~S}$ that accumulate in the $\operatorname{rrp} 6 \Delta$ or Rrp6p-anchor away strains (marked as $5.8 \mathrm{~S}$ $+30 \mathrm{~S} / \mathrm{L}$ on Fig. 4C) and correspond to the same species detected in strains in which core components of the exosome are inactivated, as shown by comparison with a strain in which Rrp43p is exported out of the nucleus (Fig. 4C). Rrp43p nuclear export was performed using the anchor away technique, by fusing Rrp43p to the FRB protein domain and treating cells with Rapamycin (Haruki et al. 2008). The pattern detected on Figure 4C was similar to the one observed when exosome subunits were depleted using conditional promoters (Allmang et al. 1999a). In conclusion, we found that all three Rrp40p mutations affect the pre-rRNA processing functions of the exosome, albeit to different extents and severity. Because the effects of the W195R mutation on rRNA processing are the most pronounced and correlate with the most severe growth defects, and because the molecular effects of the G8A and G148C mutants can only be detected on rRNA processing, we conclude that the most significant impact of the neurological mutations when introduced into Rrp40p is on the processing of the pre-rRNA precursor.

A recently published study also analyzed in S. cerevisiae the impact of some of the same mutations analyzed here (Fasken et al. 2016). Our results are consistent with most of the results reported in this study, including the effects on growth. Interestingly, this study showed that the W195R mutation results in an unstable protein, which does not associate well with the exosome complex and is degraded by the proteasome, especially when coexpressed with the wild-type version of Rrp40p (Fasken et al. 2016). This result suggests that the growth and RNA processing phenotypes of the W195R mutant are due to destabilization of the Rrp40p/ EXOSC3 protein, leading to a fraction of the exosome complexes lacking this subunit, which might potentially destabilize the complex or lack the ability to associate with RNA (Fig. 1B).

One of the major differences between our results and those reported in this study is that the authors did not find any impact of these mutations on pre-rRNA processing, as no accumulation of the ITS- 2 containing pre-rRNA species was detected by RT-PCR for the two mutants analyzed, G8A and W195R (Fasken et al. 2016). In contrast, our analysis 
clearly shows that some of the Rrp40p mutations significantly impact pre-rRNA processing, with a large accumulation of some precursors, intermediates, and aberrant forms for the W195R mutant, and a more subtle but detectable phenotype for the two other mutants (Fig. 4). We believe that this discrepancy may have been caused by the primer set used by Fasken et al. (2016) in their RT-PCR analysis. The reverse primer used in this study is positioned just downstream from the C2 cleavage site in the ITS2 (Supplemental Fig. S2) and is therefore not appropriate to amplify the 7S pre-rRNA and shorter processing products (we also note that there is a switch between the forward and reverse primers in Supplemental Table 2). This difference is sufficient to explain the fact that Fasken et al. (2016) did not detect an accumulation of the 7S species in their mutants, leading to a similar ITS2 signal for all strains analyzed.

The findings that we describe here in yeast are reminiscent of the clinical manifestations in patients who harbor different mutations affecting the EXOSC 3 gene. Among the three mutations tested, the W238R (S. cerevisiae W195R) is associated with the most severe clinical phenotype, correlating with the severe growth defect described here in yeast. This genotype was found in a sib pair compound heterozygous for the G31A substitution, presenting with congenital profound cerebellar hypoplasia with hypotonia and global developmental delay, dying by age 7-8 mo (Wan et al. 2012; RudnikSchöneborn et al. 2013). The G31A mutation (c.92G >C genotype) was first discovered in patients who presented with congenital cerebellar hypoplasia with spinal motor neuron involvement and death during infancy (Wan et al. 2012). This mutation was subsequently identified in additional patients of Czech Roma origin (Schwabova et al. 2013; Eggens et al. 2014). Patients homozygous for the c.92G > C;p.G31A mutation rarely survive infancy, with survival ranging up to 17 mo (Schwabova et al. 2013; Eggens et al. 2014). Finally, the G191C mutation (genotype c.571G > T) was associated with the mildest clinical features with the least cognitive impairment and the longest survival. Homozygous c.571G > T;p.Gly191Cys was identified in two sib pairs in a consanguineous family who presented with progressive spastic paraplegia of childhood onset, reflecting upper motor neuron involvement with survival into adulthood in the setting of mild cerebellar hypoplasia without spinal motor neuron disease (Halevy et al. 2014).

Interestingly, analysis of $5.8 \mathrm{~S}$ rRNA processing in human fibroblasts from a subject in a family carrying a D132A mutation in EXOSC3 did not reveal any significant accumulation of the 7S intermediate (Wan et al. 2012). It is possible that this mutation, which correlates with a relatively mild phenotype, might not be sufficiently damaging to result in any processing defect in normal conditions. Based on the results presented in this study and on the similarities between the severity of the phenotypes of the mutations in Rrp40p and $\mathrm{EXOSC} 3$ and on this previous analysis, we propose that mutations of EXOSC 3 that result in neurological diseases

affect rRNA processing and that the severity of processing defects is correlated with that of the growth defects in yeast and of the pathology in human patients. It remains to be established why cerebellar and motor neurons are particularly prone to defects in exosome function.

\section{MATERIALS AND METHODS}

Exosome mutants were generated using the CRISPRm system in a BY4742 background strain. pCAS plasmids containing specific sgRNAs proximal to each missense mutation were constructed using phosphorylated primers, as previously described (Stovicek et al. 2015). BY4742 cells were transformed with $1 \mu \mathrm{g}$ of pCAS plasmid and $5 \mu \mathrm{g}$ of 60 -mer homologous DNA harboring the desired missense mutations as previously described (Ryan et al. 2014). Transformants were plated on YPD $+\mathrm{G} 418$ and grown at $37^{\circ} \mathrm{C}$ for two nights and then transferred to $30^{\circ} \mathrm{C}$. Individual colonies were patched on YPD plates and checked by PCR and sequencing. For liquid culture growth, yeast strains were grown to exponential phase in YPD and then back-diluted in YPD to OD $=0.10$ at $t=0$. Cell densities were measured approximately every $30 \mathrm{~min}$ thereafter for $3 \mathrm{~h}$. Northern blot analysis and quantitation of signals were performed as described in Gabunilas and Chanfreau (2016). Most Northern blots were hybridized with $5^{\prime}$-end labeled oligonucleotide probes listed below, with the exception of the RPL18B blots, which were hybridized with an antisense RNA probe generated from a PCR product obtained with the RPL18B T3 FW/RV primers listed below.

U4 3' ext:

U4 mature:

tRNA Leu mature:

tRNA Leu intron-mature hybrid probe:

RPL18B T3 Fwd:

RPL18B T3 Rev:

1663 (ITS1):

1660 (ITS2):

020 (ITS2):

5.8S rRNA Probe:

5S rRNA Probe:

\section{CAGTCCCTTTGAAAGAATGAAT CGGACGAATCCTCACTGATA GGCGCCTGATTCAAGCTCAGGTA TCGTAAG}

CGATACCTGAGTATTCCCAC

TATTAGGTTGGGAAAGAGGG AATTAACCCTCACTAAAGGGAGG CACCAAAGGTATATGGTGTGGG CTCTTGTCTTCTTGCCCAGTAAAAG AGGCCAGCAATTTCAAGTTAACTCC TGAGAAGGAAATGACGCT CCAAGGGGCGCAATGTGCGTTCA AAGATTCGATGA CTCGGTCAGGCTCTTACCAGCTTA ACTACA

\section{SUPPLEMENTAL MATERIAL}

Supplemental material is available for this article.

\section{ACKNOWLEDGMENTS}

This work was supported by grant R01 GM61518 from the National Institute of General Medical Sciences (NIGMS) to G.C. J.G. was supported by the NIGMS training program T32GM007185. We thank Kevin Roy for discussions and one anonymous reviewer for a helpful suggestion.

Received November 15, 2016; accepted December 22, 2016. 


\section{REFERENCES}

Allmang C, Kufel J, Chanfreau G, Mitchell P, Petfalski E, Tollervey D. 1999a. Functions of the exosome in rRNA, snoRNA and snRNA synthesis. EMBO J 18: 5399-5410.

Allmang C, Petfalski E, Podtelejnikov A, Mann M, Tollervey D, Mitchell P. 1999b. The yeast exosome and human PM-Scl are related complexes of 3' $\rightarrow 5^{\prime}$ exonucleases. Genes Dev 13: 2148-2158.

Allmang C, Mitchell P, Petfalski E, Tollervey D. 2000. Degradation of ribosomal RNA precursors by the exosome. Nucleic Acids Res 28: 1684-1691.

Bousquet-Antonelli C, Presutti C, Tollervey D. 2000. Identification of a regulated pathway for nuclear pre-mRNA turnover. Cell 102: 765-775.

Egecioglu DE, Henras AK, Chanfreau GF. 2006. Contributions of Trf4pand Trf5p-dependent polyadenylation to the processing and degradative functions of the yeast nuclear exosome. RNA 12: 26-32.

Eggens VR, Barth PG, Niermeijer J-MF, Berg JN, Darin N, Dixit A, Fluss J, Foulds N, Fowler D, Hortobágyi T, et al. 2014. EXOSC3 mutations in pontocerebellar hypoplasia type 1: novel mutations and genotype-phenotype correlations. Orphanet J Rare Dis 9: 23.

Fasken MB, Losh JS, Leung SW, Brutus S, Avin B, Vaught JC, PotterBirriel J, Craig T, Tsanova B, Conn GL, et al. 2016. Insight into the RNA exosome complex through modeling pontocerebellar hypoplasia type 1b disease mutations in yeast. Genetics 12: 26-32.

Gabunilas J, Chanfreau G. 2016. Splicing-mediated autoregulation modulates Rpl22p expression in Saccharomyces cerevisiae. PLoS Genet 12: e1005999.

Gudipati RK, Xu Z, Lebreton A, Seraphin B, Steinmetz LM, Jacquier A, Libri D. 2012. Extensive degradation of RNA precursors by the exosome in wild-type cells. Mol Cell 48: 409-421.

Halevy A, Lerer I, Cohen R, Kornreich L, Shuper A, Gamliel M, El Zimerman B, Korabi I, Meiner V, Straussberg R, et al. 2014. Novel EXOSC3 mutation causes complicated hereditary spastic paraplegia. J Neurol 261: 2165-2169.

Haruki H, Nishikawa J, Laemmli UK. 2008. The anchor-away technique: rapid, conditional establishment of yeast mutant phenotypes. Mol Cell 31: 925-932.

Kadaba S, Krueger A, Trice T, Krecic AM, Hinnebusch AG, Anderson J. 2004. Nuclear surveillance and degradation of hypomodified initiator tRNA ${ }^{\mathrm{Met}}$ in S. cerevisiae. Genes Dev 18: 1227-1240.

Lebreton A, Tomecki R, Dziembowski A, Séraphin B. 2008. Endonucleolytic RNA cleavage by a eukaryotic exosome. Nature 456: 993-996.

Lubas M, Andersen PR, Schein A, Dziembowski A, Kudla G, Jensen TH. 2015. The human nuclear exosome targeting complex is loaded onto newly synthesized RNA to direct early ribonucleolysis. Cell Rep 10: 178-192.

Makino DL, Baumgärtner M, Conti E. 2013. Crystal structure of an RNA-bound 11-subunit eukaryotic exosome complex. Nature 495: $70-75$.

Makino DL, Schuch B, Stegmann E, Baumgärtner M, Basquin C, Conti E. 2015. RNA degradation paths in a 12-subunit nuclear exosome complex. Nature 524: 54-58.

Mitchell P, Petfalski E, Shevchenko A, Mann M, Tollervey D. 1997. The exosome: a conserved eukaryotic RNA processing complex containing multiple $3^{\prime} \rightarrow 5^{\prime}$ exoribonucleases. Cell 91: 457-466.
Pefanis E, Wang J, Rothschild G, Lim J, Kazadi D, Sun J, Federation A, Chao J, Elliott O, Liu Z-P, et al. 2015. RNA exosome-regulated long non-coding RNA transcription controls super-enhancer activity. Cell 161: 774-789.

Rudnik-Schöneborn S, Senderek J, Jen JC, Houge G, Seeman P, Puchmajerová A, Graul-Neumann L, Seidel U, Korinthenberg R, Kirschner J, et al. 2013. Pontocerebellar hypoplasia type 1: clinical spectrum and relevance of EXOSC3 mutations. Neurology 80: $438-446$.

Ryan OW, Skerker JM, Maurer MJ, Li X, Tsai JC, Poddar S, Lee ME, DeLoache W, Dueber JE, Arkin AP, et al. 2014. Selection of chromosomal DNA libraries using a multiplex CRISPR system. Elife 3: e03703.

Sayani S, Chanfreau GF. 2012. Sequential RNA degradation pathways provide a fail-safe mechanism to limit the accumulation of unspliced transcripts in Saccharomyces cerevisiae. RNA 18: 1563-1572.

Schaeffer D, Tsanova B, Barbas A, Reis FP, Dastidar EG, SanchezRotunno M, Arraiano CM, van Hoof A. 2009. The exosome contains domains with specific endoribonuclease, exoribonuclease and cytoplasmic mRNA decay activities. Nat Struct Mol Biol 16: 56-62.

Schneider C, Leung E, Brown J, Tollervey D. 2009. The N-terminal PIN domain of the exosome subunit Rrp44 harbors endonuclease activity and tethers Rrp44 to the yeast core exosome. Nucleic Acids Res 37: $1127-1140$.

Schwabova J, Brozkova DS, Petrak B, Mojzisova M, Pavlickova K, Haberlova J, Mrazkova L, Hedvicakova P, Hornofova L, Kaluzova M, et al. 2013. Homozygous EXOSC3 mutation c. $92 \mathrm{G} \rightarrow$ C, p.G31A is a founder mutation causing severe pontocerebellar hypoplasia type 1 among the Czech Roma. J Neurogenet 27: 163-169.

Stovicek V, Borodina I, Forster J. 2015. CRISPR-Cas system enables fast and simple genome editing of industrial Saccharomyces cerevisiae strains. Metab Eng Commun 2: 13-22.

van Hoof A, Lennertz P, Parker R. 2000. Yeast exosome mutants accumulate $3^{\prime}$-extended polyadenylated forms of U4 small nuclear RNA and small nucleolar RNAs. Mol Cell Biol 20: 441-452.

van Hoof A, Frischmeyer PA, Dietz HC, Parker R. 2002. Exosome-mediated recognition and degradation of mRNAs lacking a termination codon. Science 295: 2262-2264.

Wan J, Yourshaw M, Mamsa H, Rudnik-Schöneborn S, Menezes MP, Hong JE, Leong DW, Senderek J, Salman MS, Chitayat D, et al. 2012. Mutations in the RNA exosome component gene EXOSC3 cause pontocerebellar hypoplasia and spinal motor neuron degeneration. Nat Genet 44: 704-708.

Wasmuth EV, Januszyk K, Lima CD. 2014. Structure of an Rrp6-RNA exosome complex bound to poly(A) RNA. Nature 511: 435-439.

Wery M, Ruidant S, Schillewaert S, Leporé N, Lafontaine DLJ. 2009. The nuclear poly(A) polymerase and exosome cofactor Trf5 is recruited cotranscriptionally to nucleolar surveillance. RNA 15: 406-419.

Wyers F, Rougemaille M, Badis G, Rousselle JC, Dufour ME, Boulay J, Regnault B, Devaux F, Namane A, Seraphin B, et al. 2005. Cryptic pol II transcripts are degraded by a nuclear quality control pathway involving a new poly(A) polymerase. Cell 121: 725-737.

Zinder JC, Wasmuth EV, Lima CD. 2016. Nuclear RNA exosome at 3.1 $\AA$ reveals substrate specificities, RNA paths, and allosteric inhibition of Rrp44/Dis3. Mol Cell 64: 734-745. 

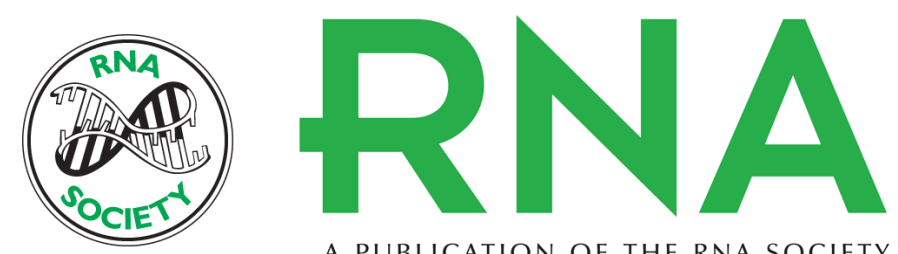

A PUBLICATION OF THE RNA SOCIETY

\section{Mutations of EXOSC3/Rrp40p associated with neurological diseases impact ribosomal RNA processing functions of the exosome in $S$. cerevisiae}

Abby Gillespie, Jason Gabunilas, Joanna C. Jen, et al.

RNA 2017 23: 466-472 originally published online January 4, 2017

Access the most recent version at doi:10.1261/rna.060004.116

Supplemental Material

References

Open Access

Creative Commons License

Email Alerting Service
http://rnajournal.cshlp.org/content/suppl/2017/01/04/rna.060004.116.DC1

This article cites 32 articles, 8 of which can be accessed free at: http://rnajournal.cshlp.org/content/23/4/466.full.html\#ref-list-1

Freely available online through the RNA Open Access option.

This article, published in RNA, is available under a Creative Commons License (Attribution 4.0 International), as described at http://creativecommons.org/licenses/by/4.0/.

Receive free email alerts when new articles cite this article - sign up in the box at the top right corner of the article or click here.

To subscribe to $R N A$ go to:

http://rnajournal.cshlp.org/subscriptions 